\title{
Ultrasonographic Examination of the Rumen in Healthy Cows
}

\author{
Sheikh Imran, Adarsh Kumar, S. P. Tyagi, Amit Kumar, and Shivali Sharma \\ Department of Veterinary Surgery and Radiology, College of Veterinary and Animal Sciences, \\ CSK HP Agricultural University, Palampur, Himachal Pradesh 176 062, India \\ Correspondence should be addressed to Sheikh Imran, sheikhimran_08@rediffmail.com
}

Received 7 January 2011; Revised 28 February 2011; Accepted 2 March 2011

Academic Editor: Margarethe Hoenig

Copyright (c) 2011 Sheikh Imran et al. This is an open access article distributed under the Creative Commons Attribution License, which permits unrestricted use, distribution, and reproduction in any medium, provided the original work is properly cited.

10 healthy Indian Jersey/Red Sindhi crossbred nonpregnant cows were subjected to transabdominal ultrasonography to develop baseline topographical data of the rumen. The wall of the rumen could be identified as a thick echogenic line adjacent to the left abdominal wall from left flank to 8 th intercostal space. The motility pattern of rumen was characterized by approximately 1 contraction every minute. The mean amplitude of the ruminal contraction was $3.2 \mathrm{~cm}$. Ultrasonography of the rumen in healthy cows is a useful adjunct to the noninvasive diagnostic investigation of the rumen.

\section{Introduction}

The rumen can be examined and evaluated using a combination of auscultation and simultaneous ballottement or percussion, by palpation through the left flank and by rectal examination. Inspection and laboratory analysis of rumen contents is also possible. Because of its large size and ease of clinical examination, rumen motility is considered to represent digestive functions in the ruminant.

It is important for the clinician to understand the motility pattern of each cycle. Specific diseases of the forestomach have characteristic alterations in motility, which aid in the diagnosis and prognosis [1]. Diagnostic ultrasonography, however, may enable the clinician to get an accurate assessment of amplitude and frequency of rumen motility in comparison to the subjective physical examination by palpation and auscultation.

Tschuor and Clauss [2] have ultrasonographically investigated the stratification of forestomach contents in ruminants. However, to our knowledge, detailed information about the ultrasonographic examination of the rumen motility pattern in healthy cows has not been reported. The present study was undertaken to assess the usefulness of ultrasonography for examining the rumen.

\section{Materials and Methods}

Ultrasonographic examinations were performed on 10 nonpregnant, clinically healthy Indian Jersey/Red Sindhi cross- bred cows. The cows were between 4 and 12 years old and weighed approximately $300-450 \mathrm{~kg}$. In order to foster proper stratification of the rumen contents, the cows were kept off feed for a period of 12 hours. The areas extending from the tuber coxae to the 6th intercostal space (ICS) and from dorsal midline to the linea alba on left side and the right paralumbar fossa were shaved. Animals were secured in standing position in a crate. The rumen and its contiguous organs were examined with a $3.5 \mathrm{MHz}$ curvilinear ultrasonic transducer. The ruminal motility frequency, that is, time interval between 2 successive peaks of ruminal contractions was observed on the display of the ultrasound machine and simultaneously recorded, by placing the transducer in the left paralumbar fossa parallel to the longitudinal axis of the cow. The amplitude of the ruminal contraction was measured electronically on the frozen ultrasonogram by means of the two cursors. The stratification of the ruminal contents was also examined ultrasonographically by moving the probe dorsoventrally either parallel to the last rib space or in the left paralumbar fossa from lumbar transverse processes to ventral midline. The rumen motility was also assessed by auscultation and palpation in the left paralumbar fossa.

\section{Results}

The wall of the rumen could be identified as a thick echogenic line $(3.0-4.8 \mathrm{~mm})$ adjacent to the left abdominal wall from left flank to the 8th ICS. The microanatomical wall layering 


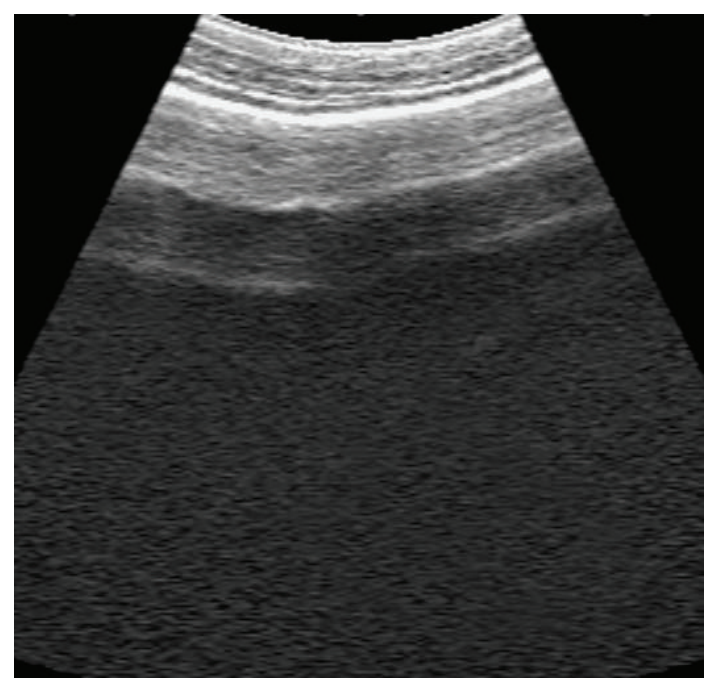

(a)

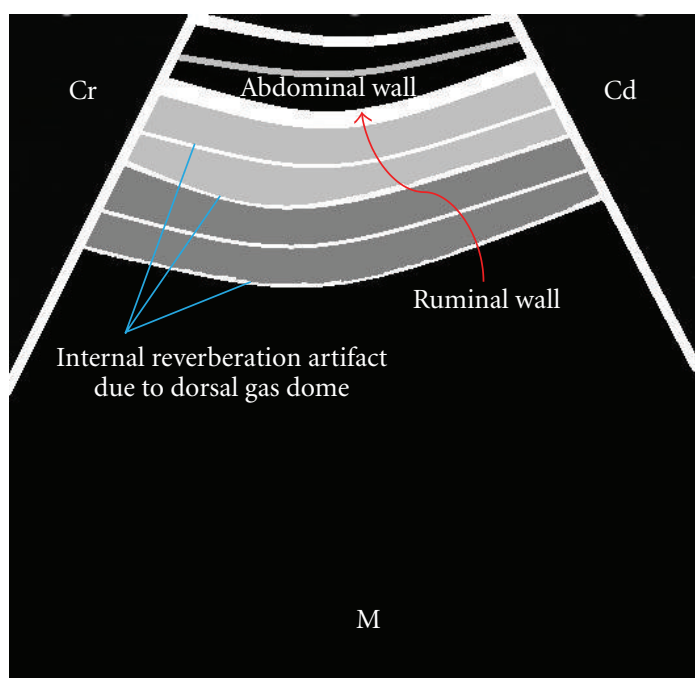

(b)

FIGURE 1: Ultrasonogram of the rumen obtained from dorsal left paralumbar fossa, by placing the transducer parallel to the longitudinal axis of the cow. Cr: cranial, Cd: caudal, and M: medial.

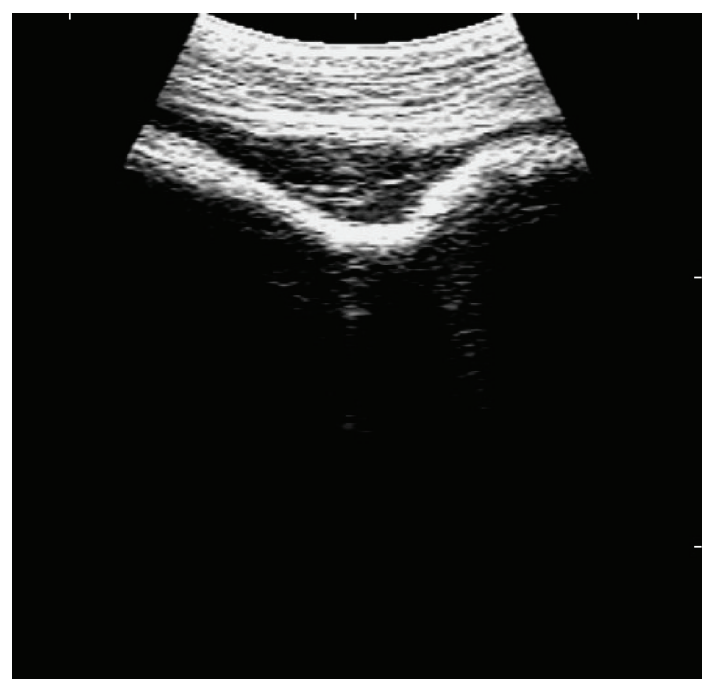

(a)

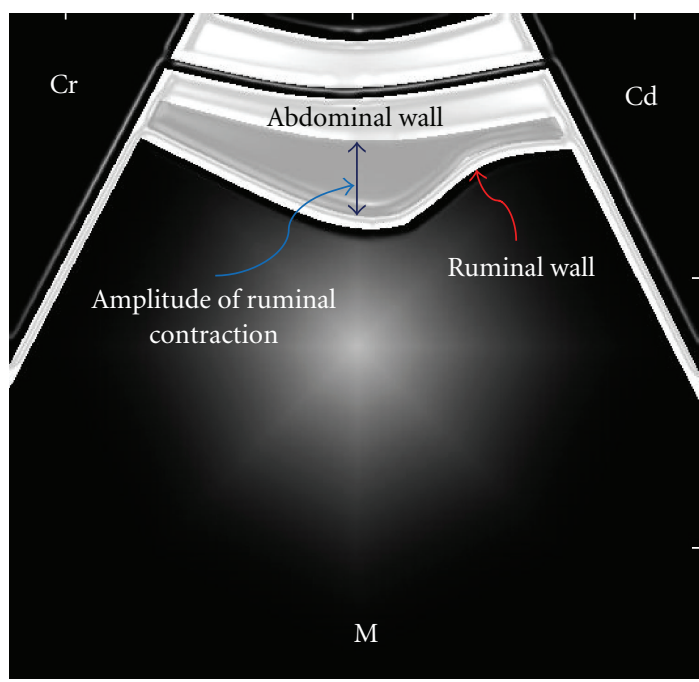

(b)

FIGURE 2: Ultrasonogram of the rumen obtained from left mid-paralumbar fossa at the peak of its contraction, by placing the transducer parallel to the longitudinal axis of the cow. Cr: cranial, Cd: caudal, and M: medial.

was indistinguishable. The gaseous zone in the most dorsal aspect of the ruminal sac could be identified by the presence of the internal reverberation artifact (Figure 1). As the transducer was moved ventral, the artifact cessated abruptly, succeeded by anechogenic to hypoechogenic ruminal ingesta interspersed with gas and fluid.

The ruminal wall contracted gradually away from the abdominal wall in a pulse-like pattern towards the cranial end (Figure 2). Subsequently, the same sequence was repeated in the opposite direction, until the ruminal wall rested against the abdominal wall in a relaxed state. The mean time interval between 2 successive peaks of ruminal contractions was (arithmetic mean \pm SD, $48.6 \pm 1.53$ ) seconds. The mean amplitude of the ruminal contraction was (arithmetic mean $\pm \mathrm{SD}, 3.2 \pm 0.1$ ) cm. On physical examination, each ruminal contraction was felt by an outward push to the clenched fist placed in the left paralumbar fossa and simultaneous auscultation of the gurgling ruminal sounds. Further, ruminal contents were characterized by a doughy consistency on palpation of the left paralumbar fossa. A comparison between ultrasonographic and physical examinations has been made in Table 1.

Other organs often seen in association with the rumen included the reticulum, abomasum, spleen, and the left 


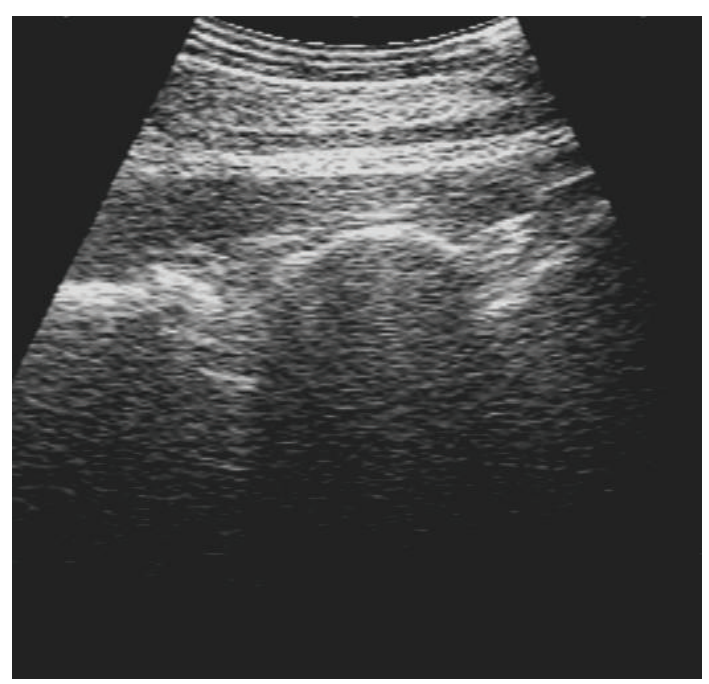

(a)

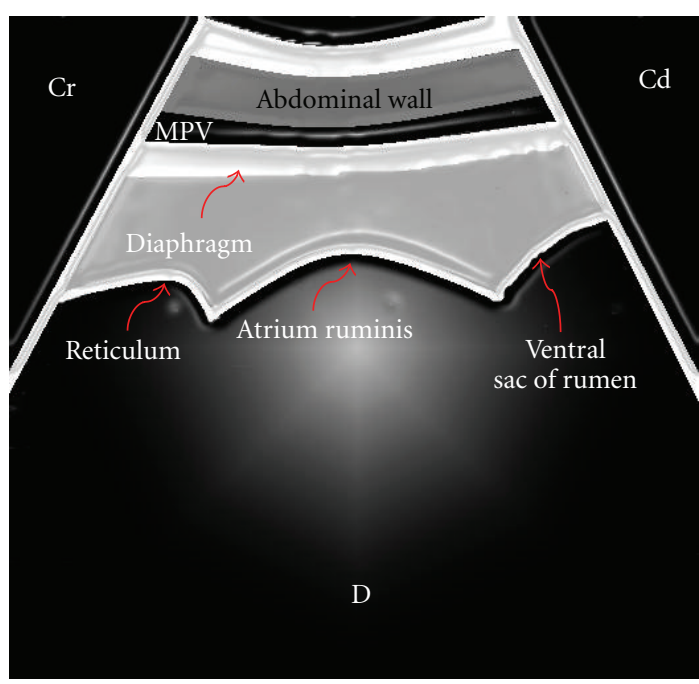

(b)

FIGURE 3: Ultrasonogram of the reticulum, atrium ruminis, and ventral sac of the rumen imaged from the left paramedian side, by placing the transducer parallel to the longitudinal axis of the cow. MPV: musculophrenic vein, Cr: Cranial, Cd: caudal, and D: dorsal.

TABLE 1: Comparison between ultrasonographic and physical examinations of the rumen.

\begin{tabular}{lcc}
\hline Checklist & Ultrasonographic examination & Physical examination \\
\hline Direct visualization and measurement of thickness of the ruminal wall & Yes & No \\
Measurement of amplitude of the ruminal contraction & Yes & No \\
Estimation of time interval between 2 successive peaks of the ruminal contractions & Yes & Yes \\
Auscultation of the ruminal sounds & No & Yes \\
Palpation of the ruminal contents & No & Yes \\
\hline
\end{tabular}

kidney. The reticulum, atrium ruminis, and part of the ventral ruminal sac were optimally seen by placing the transducer parallel to the longitudinal axis, in left ventral median or paramedian positions in only 3 cows (Figure 3 ).

In the remaining 7 cows, only reticulum, atrium ruminis, and spleen could be seen. The spleen was always seen lateral to the rumen in the 12th to 8 th ICS in all the cows. The left kidney could not be seen from the left paralumbar fossa but was imaged from the right flank in 6 cows. The motile wall of the rumen was seen medial to the left kidney in the longitudinal/sagittal imaging plane; however, it could not be observed along with the right kidney (Figure 4).

\section{Discussion}

The microanatomical wall layering of rumen was not observed. It appears that the attenuation of the ultrasound waves while traversing the abdominal wall and rumen contents resulted in poor details of its wall layering and underlying structures. Reverberation artifact observed in the dorsal sac of the rumen was the production of multiple echoes from one ultrasound pulse bouncing back and forth, created by the dorsal ruminal gas cap, which acted as a reflector. This artifact is characterized by the formation of several hyperechoic lines that are equally spaced and gradually attenuated [3].
The ultrasonographic differences between the middle ingesta and most fluid ventral phases could not be discerned in the present study. However, Tschour and Clauss [2] could detect these differences in 2 of the 3 Swiss Braunvieh cows. They reported that the middle hypoechogenic ingesta phase could be differentiated from the ventral anechogenic fluid phase by observing a change in the gradient of echogenicity of the ruminal contents. Their observations were facilitated by manual observations during ultrasonography, as the whole procedure was undertaken after performing the ruminal cannulation, whereas the present study was completely noninvasive.

In the present study, the ultrasonographic assessment of the ruminal motility (amplitude and frequency) proved to be a straightforward procedure in all the cows. Prior to ultrasonography, the motility pattern of the forestomachs was assessed from continuous electromyographic or mechanical records obtained in conscious animals [4]. However, these procedures are painful from the ethical perspective and relatively difficult to perform. In contrast, ultrasonographic examination is noninvasive and legible. Functionally, rumen movements can be classified into primary cycles, secondary cycles, and regurgitation movements. The primary cycle mixes the ingesta within the rumen and occurs every 1 to 2 minutes. The secondary cycle results in eructation and occurs 


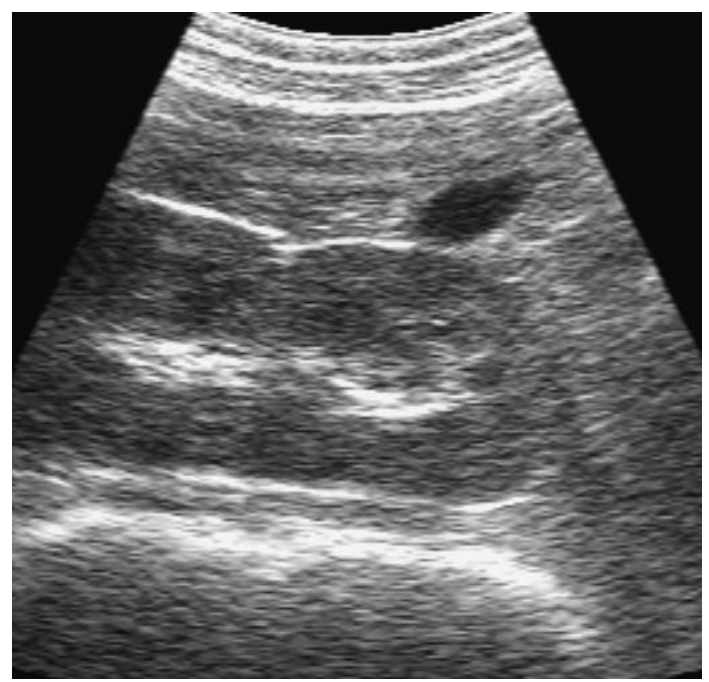

(a)

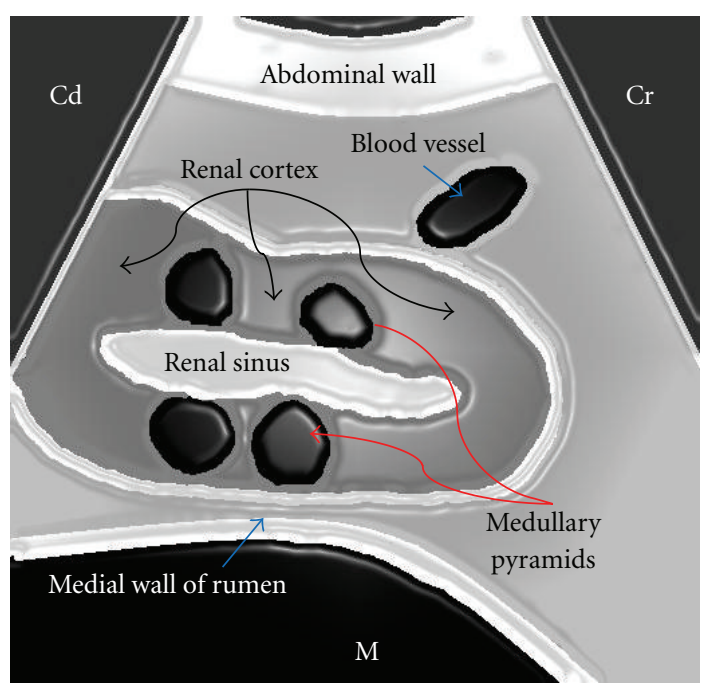

(b)

FIGURE 4: Ultrasonogram of the left kidney imaged from the right paralumbar fossa, by placing the transducer parallel to the longitudinal axis of the cow, just below the transverse processes of the lumbar vertebrae 3-5. Cr: cranial, Cd: caudal, and M: medial.

at intervals of 1 to 2 minutes. Regurgitation results from an additional ruminal contraction interposed between normal mixing movements of the rumen [5]. However, in our study, we did not hold the clue to differentiate between the primary, secondary, and regurgitation ruminal contractions.

The medial motile wall of the rumen was seen contiguous with the left kidney by placing the transducer in the dorsocaudal aspect of the right dorsal paralumbar fossa. Transabdominal imaging of the left kidney was facilitated by bringing it closer to the right flank, with the help of transrectal manipulation. The left kidney in the bovine is pushed to the right side by the rumen. It is almost completely surrounded by peritoneum, and therefore pendulous and lies ventral to lumbar vertebrae $2-5$ and caudal to the right kidney, from which it is separated by the descending mesocolon. Medially, it joins the rumen and, laterally, the intestinal mass [6]. Occasionally, in calves and small or thin adult cattle, the left kidney can be visible from the right caudal paralumbar fossa, but it is frequently covered by gasfilled large intestine [7]. In our opinion, low body weight was the possible reason for relatively easy imaging of the left kidney via right paralumbar fossa.

Changes in the rumen motility are indicators of disease. Hypomotility (less than 1 movement every 2 minutes) or rumen stasis may cause a free-gas bloat and be associated with a number of conditions including milk fever, ruminal acidosis and painful conditions of the abdomen. Hypermotility (more than 5 movements every 2 minutes) is less common and conditions include the development of frothy bloat, vagal indigestion and Johne's disease [5]. Our measurements are likely to be most useful for the clinicians to assess whether the motility pattern (amplitude and frequency) of the rumen is normal or not.

It can be concluded that ultrasonography is a useful adjunct to the physical examination for the noninvasive diagnostic investigation of the rumen.

\section{References}

[1] O. M. Radostits, C. C. Gay, D. C. Blood, and K. W. Hinchcliff, "Diseases of the alimentary tract II," in Veterinary Medicine: A Textbook of the Diseases of Cattle, Horses, Sheep, Pigs and Goats, pp. 293-294, W. B. Saunders, Philadelphia, Pa, USA, 10th edition, 2007.

[2] A. Tschuor and M. Clauss, "Investigations on the stratification of forestomach contents in ruminants: an ultrasonographic approach," European Journal of Wildlife Research, vol. 54, no. 4, pp. 627-633, 2008.

[3] L. Blond and S. Buczinski, "Basis of ultrasound imaging and the main artifacts in bovine medicine," Veterinary Clinics of North America-Food Animal Practice, vol. 25, no. 3, pp. 553-565, 2009.

[4] Y. Ruckebusch and T. Tomov, "The sequential contractions of the rumen associated with eructation in sheep," Journal of Physiology, vol. 235, no. 2, pp. 447-458, 1973.

[5] P. G. G. Jackson and P. D. Cockcroft, Clinical Examination of Farm Animals, Blackwell Science, 1st edition, 2002.

[6] K. D. Budras, R. E. Habel, A. Wünsche, and S. Buda, Bovine Anatomy, Schlütersche $\mathrm{GmbH}$, 1st edition, 2003.

[7] M. Floeck, "Ultrasonography of bovine urinary tract disorders," Veterinary Clinics of North America-Food Animal Practice, vol. 25, no. 3, pp. 651-667, 2009. 

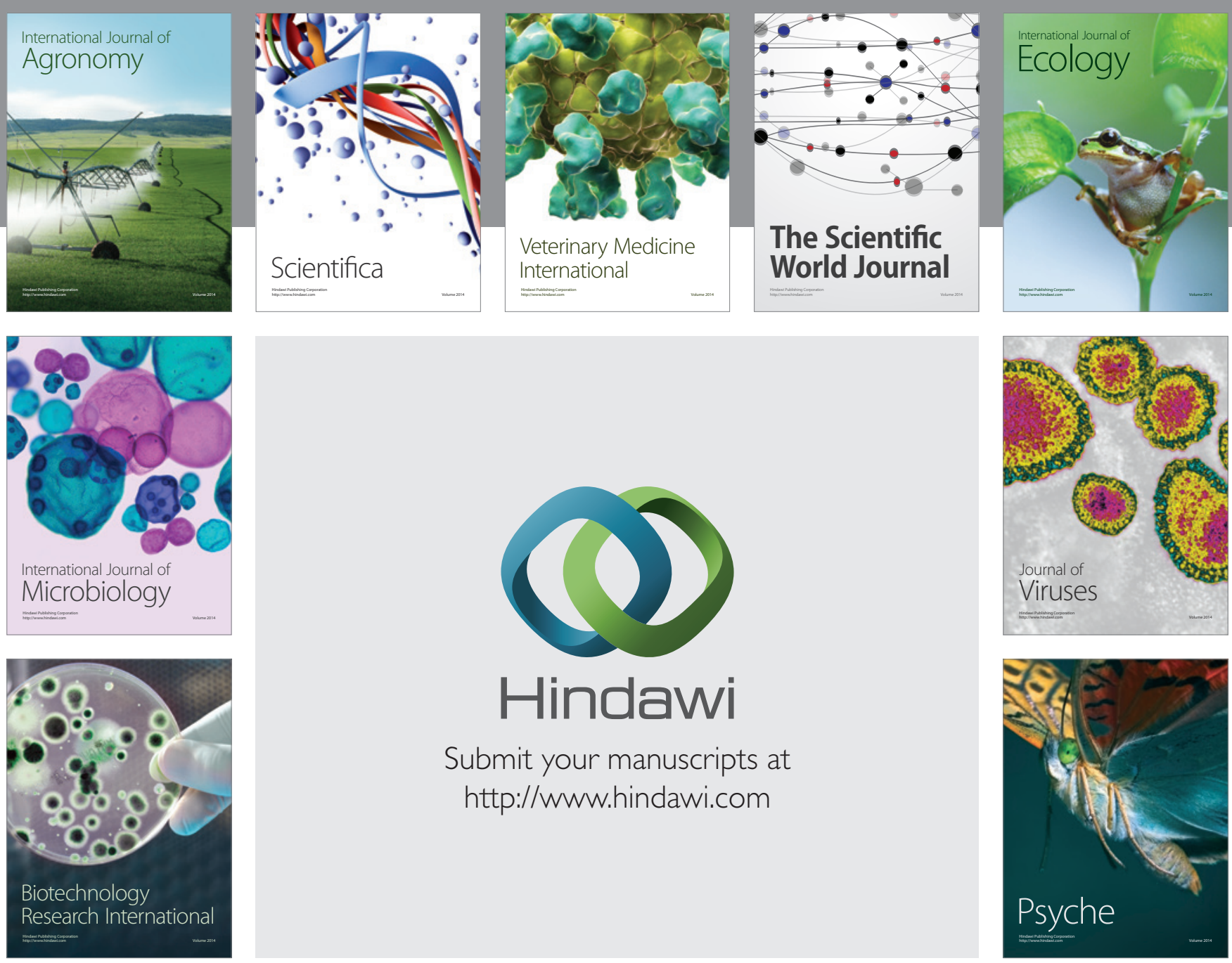

Submit your manuscripts at

http://www.hindawi.com
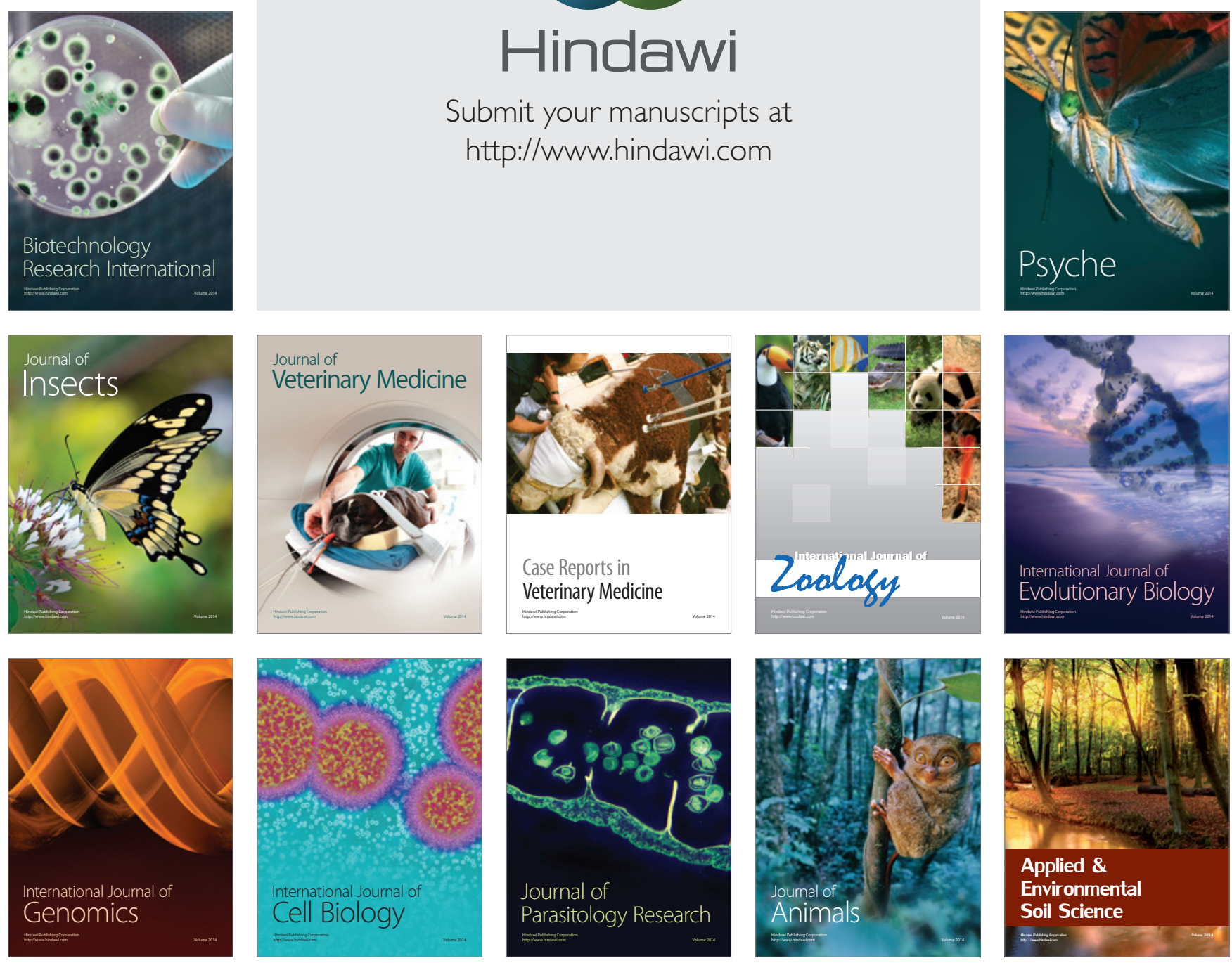